\title{
Classification of Swimming Microorganisms Motion Patterns in 4D Digital In-Line Holography Data
}

\author{
Laura Leal-Taixé ${ }^{1, \star}$, Matthias Heydt, Sebastian Weiße ${ }^{2}$, Axel Rosenhahn ${ }^{2,3}$, \\ and Bodo Rosenhahn ${ }^{1}$ \\ ${ }^{1}$ Leibniz Universität Hannover, Appelstr. 9A, Hannover, Germany \\ 2 Applied Physical Chemistry, University of Heidelberg, INF 253, Heidelberg, Germany \\ ${ }^{3}$ Institute of Toxicology and Genetics (ITG), Forschungszentrum Karlsruhe, Germany \\ leal@tnt. uni-hannover. de
}

\begin{abstract}
Digital in-line holography is a 3D microscopy technique which has gotten an increasing amount of attention over the last few years in the fields of microbiology, medicine and physics. In this paper we present an approach for automatically classifying complex microorganism motions observed with this microscopy technique. Our main contribution is the use of Hidden Markov Models (HMMs) to classify four different motion patterns of a microorganism and to separate multiple patterns occurring within a trajectory. We perform leave-oneout experiments with the training data to prove the accuracy of our method and to analyze the importance of each trajectory feature for classification. We further present results obtained on four full sequences, a total of 2500 frames. The obtained classification rates range between $83.5 \%$ and $100 \%$.
\end{abstract}

\section{Introduction}

Many fields of interest in biology and other scientific research areas deal with intrinsically three-dimensional problems. The motility of swimming microorganisms such as bacteria or algae is of fundamental importance for topics like pathogen-host interactions [1], biofilm-formation [2], or biofouling by marine microorganisms [3].

Understanding the motility and behavioral patterns of microorganisms allows us to understand their interaction with the environment and thus to control environmental parameters to avoid unwanted consequences such as infections or biofouling. To study these effects in 3D several attempts have been made: tracking light microscopy, capable of tracking one bacterium at a time [4], stereoscopy [5] or confocal microscopy [6].

Fully automated analyzing tools are becoming necessary given the huge amount of data that can be obtained with these imaging techniques. Besides generating motion trajectories from microscopic data, a classification afterwards allows the biologists to get in a compact and compressed fashion the desired information from the large image sets. Indeed, the classification of motion patterns in biology is a well-studied topic [7] but identifying these patterns manually is a complicated task. Recently, machine learning and pattern recognition techniques have been introduced to analyze in detail such complex movements. These techniques include: Principal Component Analysis (PCA) [8],

\footnotetext{
* Corresponding author.

M. Goesele et al. (Eds.): DAGM 2010, LNCS 6376, pp. 283-292 2010.

(C) Springer-Verlag Berlin Heidelberg 2010
} 


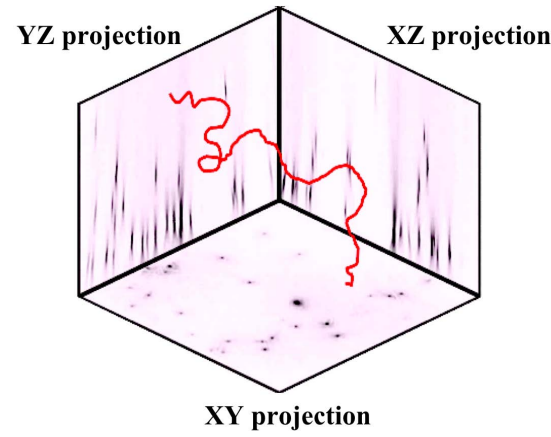

(a)

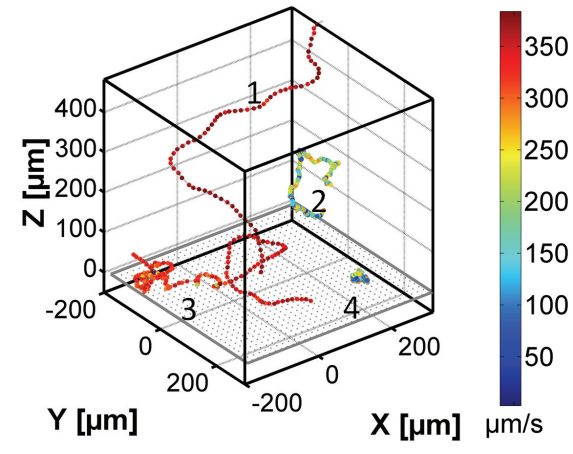

(b)

Fig. 1. (a) Projections obtained with digital in-line holography (inverted colors for better visualization). Sample trajectory in red. (b) Four patterns colored according to speed: orientation (1), wobbling (2), gyration (3) and intensive surface probing (4)

a linear transformation used to analyze high dimensional data; Bayesian models [9] which use a graph model and the rules of probability theory to select among different hypotheses; or Support Vector Machines (SVM) [10], which use training data to find the optimum parameters of the model representing each class. A comparison of machine learning approaches applied to biology can be found in [11].

In order to classify biological patterns, we need to use an approach able to handle time-varying signals. Hidden Markov Models [12] are statistical models especially known for their application in temporal pattern recognition. They were first used in speech recognition and since then, HMMs have been extensively applied to vision. Applications vary from handwritten word recognition [13], face recognition [14] or human action recognition [15, 16].

In this paper, we focus on the classification of four motion patterns of the green alga Ulva linza with the use of Hidden Markov Models. Furthermore, our system is able to find and separate different patterns within a single sequence. Besides classification of motion patterns, a key issue is the choice of features used to classify and distinguish the involved patterns. For this reason we perfom an extensive analysis of the importance of typical motion parameters, such as velocity, curvature, orientation, etc. Our developed system is highly flexible and can easily be extended. Especially for forthcoming work on cells, microorganisms or human behavior, such automated algorithms are of pivotal importance for high throughput analysis of individual segments in motion data.

\section{Digital in-line Holography (DIH)}

An alternative to conventional optical microscopy is provided by digital in-line holography, a lensless microscopy technique which intrinsically contains three-dimensional information about the volume under investigation.

The important requirements are a sample of sufficient transparency and a divergent, coherent wave. The holographic microscope setup follows directly Gabors initial idea [17] and has been implemented for laser radiation by $\mathrm{Xu}$ et al. [18]. A divergent 
wavefront is generated by diffraction of a laser beam at a pinhole. A so-called hologram is then recorded by a CCD- or CMOS-chip. This hologram is then reconstructed back into real space by a Kirchhoff-Helmholtz transformation [18]:

$$
K(\mathbf{r})=\int_{S} I(\xi) \exp \left[\frac{i k \mathbf{r} \xi}{|\xi|}\right] d \xi
$$

The integration extends over the 2D surface of the screen with coordinates $\xi=(X, Y, L)$, where $L$ is the distance from the source (pinhole) to the center of the detector (CCD chip), $I(\xi)$ is the contrast image (hologram) on the screen obtained by subtracting the images with and without the object present and $k$ the wave number: $k=2 \pi / \lambda$.

From the reconstruction 3 projections $X Y, X Z$ and $Y Z$ are obtained (see Figure 1(a) as described in [19]. These projections contain the image information of the complete observation volume, i.e. from every object located in the light cone between pinhole and detector. The extraction of the particle coordinates in combination with a time series of holograms enables one to track multiple objects in 3D over time [3, 20].

Determining 3D trajectories is a complex task which still presents some major challenges. We make use of the system described in [21], which uses the multi-level Hungarian to obtain the full 3D trajectories from 3 projections. Trajectories are then manually verified by specialists and tagged according to their motion pattern.

\section{Hidden Markov Models}

Hidden Markov Models [12] are statistical models of sequencial data widely used in many applications in artificial intelligence, speech and pattern recognition and modeling of biological sequences.

In an HMM it is assumed that the system being modeled is a Markov process with unobserved states. This hidden stochastic process can only be observed through another set of stochastic processes that produce the sequence of symbols $O=o_{1}, o_{2}, \ldots, o_{M}$. An HMM consists of a number $N$ of states $S_{1}, S_{2}, \ldots, S_{N}$. The system is at one of the states at any given time. Every HMM can be defined by the triple $\lambda=(\Pi, A, B)$. $\Pi=\left\{\pi_{i}\right\}$ is the vector of initial state probabilities. Each transition from $S_{i}$ to $S_{j}$ can occur with a probability of $a_{i j}$, where $\sum_{j} a_{i j}=1 . A=\left\{a_{i j}\right\}$ is the state transition matrix. In addition, each state $S_{i}$ generates an output $o_{k}$ with a probability distribution $b_{i k}=P\left(o_{k} \mid S_{i}\right) . B=\left\{b_{i k}\right\}$ is the emission matrix.

A detailed introduction to HMM theory can be found in [12].

\section{HMMs for Motion Pattern Classification}

In this section we describe the different types of motion patterns to classify, as well as the design of the complete HMM and the features used for classification.

\subsection{Types of Patterns}

In our experimental setup we are interested in the four patterns depicted in Figure 1(b) Orientation(1), Wobbling(2), Gyration(3) and intensive surface probing or Spinning(4). 
They show a high similarity with the patterns observed before in [22] for the brown algae Hincksia Irregularis.

Orientation. Pattern 1 in Figure 1(b) is an example for the Orientation pattern. This pattern typically occurs in solution and far away from surfaces. The most important characteristics of the pattern are the high swimming speed (a mean of $150 \mu \mathrm{m} / \mathrm{s}$ ) and a straight swimming motion with moderate turning angles.

Wobbling. Pattern 2 is called the Wobbling pattern and its main characteristic is a much slower mean velocity of around $50 \mu \mathrm{m} / \mathrm{s}$. The spores assigned to the pattern often change their direction of movement and only swim in straight lines for very short distances. Compared to the orientation pattern this leads to less smooth trajectories.

Gyration. Pattern 3 is called the Gyration pattern. This pattern is extremely important for the exploration of surfaces as occasional surface contacts are observable. The behavior in solution is similar to the Orientation pattern. Since in this pattern spores often switch between swimming towards and away from the surfaces, it can be interpreted as a pre-stage to surface probing.

Intensive surface probing and Spinning. Pattern 4 involves a swimming in circles close to the surface within a very limited region. After a certain exploration time, the spores leave the surface to the next position and start swimming in circular patterns again. This motion is characterized by decreased mean velocities of about $30 \mu \mathrm{m} / \mathrm{s}$ in combination with a higher tendency to change direction (see Figure 1(b), case 4).

\subsection{Features Used for Classification}

An analysis of the features used for classification is presented in this section. Many of the features are typical and can be used in any motion analysis problem. An intrinsic characteristic of digital in-line holography is the lower resolution of the $z$ position compared to the $x, y$ resolution [23]. Since many of the following features depend on the depth value, we compute the average measurements within 5 frames in order to reduce the noise of such features. The four characteristic features used are:

- $v$, velocity: the speed of the particles is an important descriptive feature as we can see in Figure 1(b) We use only the magnitude of the speed vector, since the direction is described by the next two parameters. Range is $[0$, maxSpeed $]$. maxSpeed is the maximum speed of the particles as found experimentally in [19].

- $\alpha$, angle between velocities: it measures the change in direction, distinguishing stable patterns from random ones. Range is $[0,180]$.

- $\beta$, angle to normal of the surface: it measures how the particles approaches the surface or how it swims above it. Range is $[0,180]$.

- $D$, distance to surface: this can be a key feature to differentiate surface-induced movements from general movements. Range is $\left(m_{z}, M_{z}\right]$, where $m_{z}$ and $M_{z}$ are the $z$ limits of the volume under study. 
In order to work with Hidden Markov Models, we need to represent the features for each pattern with a fixed set of symbols. The number of total symbols will depend on the number of symbols used to represent each feature $N_{\text {symbols }}=N_{v} N_{\alpha} N_{\beta} N_{D}$.

In order to convert every symbol for each feature into a unique symbol for the HMM, we use Equation (2), where $Y$ is the final symbol we are looking for, $Y_{1 . .4}$ are the symbols for each of the features, ranged [1... $\left.N_{Y_{1} . .4}\right]$, where $N_{Y_{1 . .4}}$ are the number of symbols per feature.

$$
Y=Y_{1}+\left(Y_{2}-1\right) N_{Y_{1}}+\left(Y_{3}-1\right) N_{Y_{1}} N_{Y_{2}}+\left(Y_{4}-1\right) N_{Y_{1}} N_{Y_{2}} N_{Y_{3}}
$$

In the next sections we present how to use the resulting symbols to train the HMMs. The symbols are the observations of the HMM, therefore, the training process gives us the probability of emitting each symbol for each of the states.

\subsection{Building and Training the HMMs}

In speech recognition, an HMM is trained for each of the phonemes of a language. Later, words are constructed by concatenating several HMMs of the phonemes that form the word. HMMs for sentences can even be created by concatenating HMMs of words, etc. We take a similar hierarchical approach in this paper. We train one HMM for each of the patterns and then we combine them into a unique Markov chain with a simple yet effective design that will be able to describe any pattern or combination of patterns. This approach can be used in any problem where multiple motion patterns are present.

Individual HMM per pattern. In order to represent each pattern, we build a Markov chain with $N$ states and we only allow the model to stay in the same state or move one state forward. Finally, from state $N$ we can also go back to state 1 . The number of states $\mathrm{N}$ is found empirically using the training data. The HMM is trained using the Baum-Welch algorithm to obtain the transition and emission matrices.

Complete HMM. The idea of having a complete HMM that represents all the patterns is that we can not only classify sequences where there is one pattern present, but sequences where the particle makes transitions between different patterns. In Figure 2(a) we can see a representation of the complete model while the design of the transition matrix is depicted in Figure 2(b), The four individual HMMs for each of the patterns are placed in parallel (blue). In order to deal with the transitions we create two special states: the START and the SWITCH state.

The START state is just created to allow the system to begin at any pattern (orange). We define $P_{\text {start }}=P_{\text {SwitchToModel }}=\frac{1-P_{\text {switch }}}{N_{P}}$ where $N_{P}$ is the number of patterns. As START does not contain any information of the pattern, it does not emit any symbol.

The purpose of the new state SWITCH is to make transitions easier. Imagine a given trajectory which makes a transition from Pattern 1 to Pattern 2. While transitioning, the features create a symbol that neither belongs to Pattern 1 nor 2 . The system can then go to state SWITCH to emit that symbol and continue to Pattern 2. Therefore,

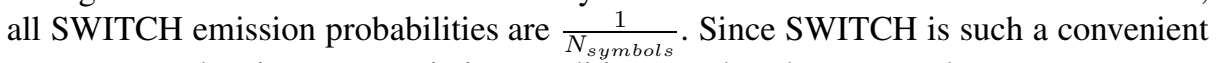
state, we need to impose restrictive conditions so that the system does not go or stay in SWITCH too often. This is controlled by the parameter $P_{\text {switch }}$, set at the minimum 


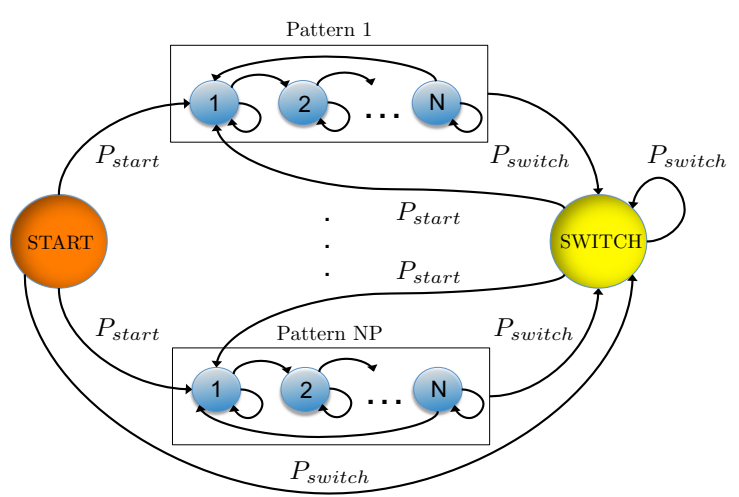

(a)

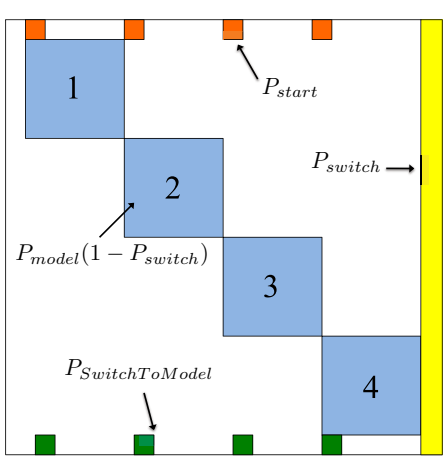

(b)

Fig. 2. (a) Complete HMM created to include changes between patterns within one trajectory. (b) Transition matrix of the complete HMM

value of all the $P_{\text {model }}$ minus a small $\epsilon$. This way, we ensure that $P_{\text {switch }}$ is the lowest transition probability in the system.

Finally, the sequence of states given by the Viterbi algorithm determines the motion pattern observed. Our implementation uses the standard MatLab HMM functions.

\section{Experimental Results}

In this section we present several experimental results to prove the use of Hidden Markov Models to classify biological patterns.

\subsection{Evaluation of the Features Used for Classification}

The experiments in this section have the purpose of determining the impact of each feature for the correct classification of each pattern. We perform leave-one-out tests on our training data which consists of 525 trajectories: 78 for wobbling, 181 for gyration, 202 for orientation and 64 for intensive surface probing. The trajectories are obtained automatically with the method in [21] and verified and classified manually by experts.

The first experiment that we conduct (see Figure 3) is to determine the effect of each parameter for the classification of all the patterns. The number of symbols and states can only be determined empirically since they depend heavily on the amount of training data. In our experiments, we found the best set of parameters to be $N=4, N_{v}=4$, $N_{\alpha}=3, N_{\beta}=3$ and $N_{D}=3$, for which we obtain a classification rate of $83.86 \%$.

For each test, we set one parameter to 1 , which means that the corresponding feature has no effect in the classification process. For example, the first bar in blue labeled "No Depth" is done with $N_{D}=1$. The classification rate for each pattern (labeled from 1 to 4) as well as the mean for all the patterns (labeled Total) is recorded. 


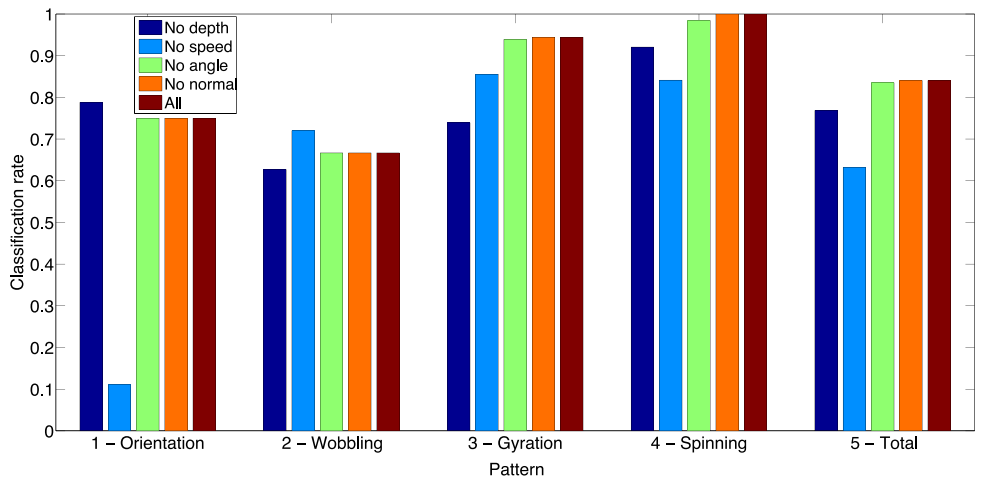

Fig. 3. Classification rate for parameters $N=4, N_{v}=4, N_{\alpha}=3, N_{\beta}=3$ and $N_{D}=3$. On each experiment, one of the features is not used. In the last experiment all features are used.

As we can see, the angle $\alpha$ and the normal $\beta$ information are the less relevant features, since the classification rate with and without these features is almost the same. The angle information depends on the $z$ component and, as explained in section 4.2 , the lower resolution in $z$ can result in noisy measurements. In this case, the trade-off is between having a noisy angle data which can be unreliable, or an average measure which is less discriminative for classification. The most distinguishing feature according to Figure 3 is the speed. Without it, the total classification rate decreases to $55.51 \%$ and down to just $11.05 \%$ for the orientation pattern.

Based on the previous results, we could think of just using the depth and speed information for classification. But if $N_{\alpha}=N_{\beta}=1$, the rate goes down to $79.69 \%$. That means that we need one of the two measures for correct classification. The parameters used are: $N=4, N_{v}=4, N_{\alpha}=1, N_{\beta}=3$ and $N_{D}=3$, for which we obtain a classification rate of $83.5 \%$. This rate is very close to the result with $N_{\alpha}=3$, with the advantage that we now use less symbols to represent the same information. Several tests lead us to choose $N=4$ number of states.

The confusion matrix for these parameters is shown in Figure 4 As we can see, patterns 3 and 4 are correctly classified. The common misclassifications occur when Orientation (1) is classified as Gyration (3), or when Wobbling (2) is classified as Spinning (4). In the next section we discuss these misclassifications in detail.

\begin{tabular}{l|cccc|}
\multicolumn{1}{l|}{} & 1 - Ori & 2 - Wob & 3 - Gyr & 4 - Spin \\
\cline { 2 - 5 } 1 - Ori & 0.75 & 0.09 & 0.16 & \\
2 - Wob & 0.07 & 0.68 & 0.01 & 0.24 \\
3 - Gyr & 0.01 & & 0.94 & 0.05 \\
4 - Spin & & & 0.02 & 0.98 \\
\cline { 2 - 4 } & & &
\end{tabular}

Fig. 4. Confusion matrix with parameters $N=4, N_{v}=4, N_{\alpha}=1, N_{\beta}=3$ and $N_{D}=3$ 


\subsection{Classification on Other Sequences}

In this section, we present the performance of the algorithm when several patterns appear within one trajectory and also analyze the typical misclassifications. As test data we use four sequences which contain 27, 40, 49 and 11 trajectories, respectively. We obtain classification rates of $100 \%, 85 \%, 89.8 \%$ and $100 \%$, respectively. Note that for the third sequence, $60 \%$ of the misclassifications are only partial, which means that the model detects that there are several patterns but only one of them is misclassified.

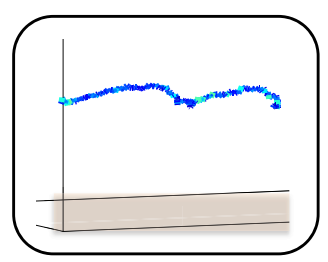

(a)

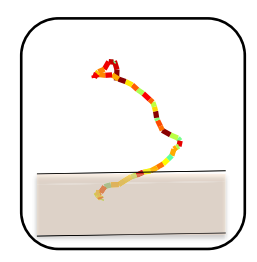

(b)

Fig. 5. (a) Wobbling (pattern 2) misclassified as Spinning (4). (b) Gyration (3) misclassified as Orientation (1). Color coded according to speed as in Figure 1(b)

One of the misclassifications that can occur is that Wobbling (2) is classified as Spinning (4). Both motion patterns have similar speed values and the only truly differentiating characteristics are the depth and the angle $\alpha$. Since we use 3 symbols for depth, the fact that the microorganism touches the surface or swims near the surface leads to the same classification. That is the case of Figure 5(a), in which the model chooses pattern Spinning (4) because the speed is very low (dark blue) and sometimes the speed in the Wobbling pattern can be a little higher (light blue).

As commented in section 4.1, Gyration (3) and Orientation (1) are two linked patterns. The behavior of gyration in solution is similar to the orientation pattern, that is why the misclassification shown in Figure 5(b) can happen. In this case, since the microorganism does not interact with the surface and the speed of the pattern is high (red color), the model detects it as an orientation pattern. We note that this pattern is difficult to classify, even for a trained expert.

On the other hand, the model has been proven to handle changes between patterns extremely well. In Figure 6(a), we see the transition between Gyration (3) and Spinning (4). In Figure 6(b) color coded according to classification, we can see how the model detects the Orientation part (red) and the Gyration part (yellow) perfectly well. The model performs a quick transition (marked in blue) and during this period the model stays in the SWITCH state. We have verified that all the transition periods detected by the model lie within the manually annotated transition boundaries marked by experts, even when there is more than one transition present in a trajectory.

The classification results on a full sequence are shown in Figure 7

Finally, we can obtain the probability of each transition (e.g. from Orientation to Spinning) for a given dataset under study. This is extremely useful for experts to understand the behavior of a certain microorganism under varying conditions. 


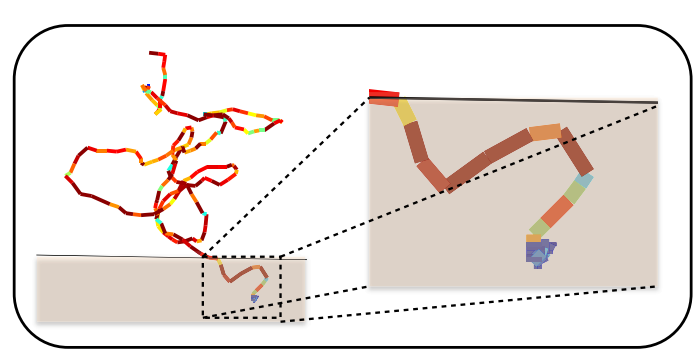

(a) Orientation (1) + Spinning (4). Zoom on the spinning part. Color coded according to speed as in Figure 1(b)

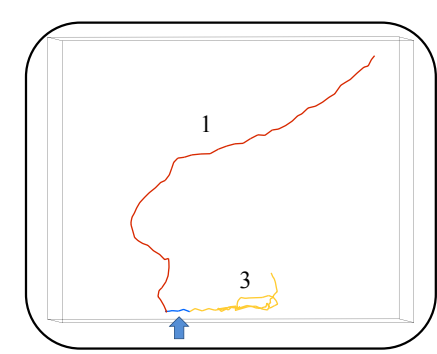

(b) Orientation $(1$, red) + Gyration (3, yellow). Transition marked in blue and pointed by an arrow.

Fig. 6. Sequences containing two patterns within one trajectory

\section{Conclusions}

We presented a fully automatic method to classify four different motion patterns of a microorganism observed with digital in-line holography. We used Hidden Markov Models for classification, since it allows us to encode the dynamic information of each pattern. We presented a simple yet effective hierarchical design which combines multiple trained HMMs (one for each of the patterns), which has proved successful to identify different patterns within one single trajectory. The changes between one pattern and another are correctly detected by the complete HMM. The experiments performed on four full sequences result in a total classification rate between $83.5 \%$ and $100 \%$. Furthermore, we presented a detailed analysis of the impact of each of the features used for classification. The proper use of a powerful machine learning tool, such as Hidden Markov Models, can be extremely useful to study microorganism motility, providing a vast amount of analyzed data to the experts.

Acknowledgements. This work is partially funded by the German Research Foundation, DFG projects RO 2497/7-1 and RO 2524/2-1 and the EU project AMBIO.

\section{References}

1. Ginger, M., Portman, N., McKean, P.: Swimming with protists: perception, motility and flagellum assembly. Nature Reviews Microbiology 6(11), 838-850 (2008)

2. Stoodley, P., Sauer, K., Davies, D., Costerton, J.: Biofilms as complex differentiated communities. Annual Review of Microbiology 56, 187-209 (2002)

3. Heydt, M., Rosenhahn, A., Grunze, M., Pettitt, M., Callow, M.E., Callow, J.A.: Digital inline holography as a $3 \mathrm{~d}$ tool to study motile marine organisms during their exploration of surfaces. The Journal of Adhesion 83(5), 417-430 (2007)

4. Frymier, P., Ford, R., Berg, H., Cummings, P.: 3d tracking of motile bacteria near a solid planar surface. Proc. Natl. Acad. Sci. U.S.A. 92(13), 6195-6199 (1995)

5. Baba, S., Inomata, S., Ooya, M., Mogami, Y., Izumikurotani, A.: $3 \mathrm{~d}$ recording and measurement of swimming paths of microorganisms with 2 synchronized monochrome cameras. Rev. of Sci. Instruments 62(2), 540-541 (1991) 


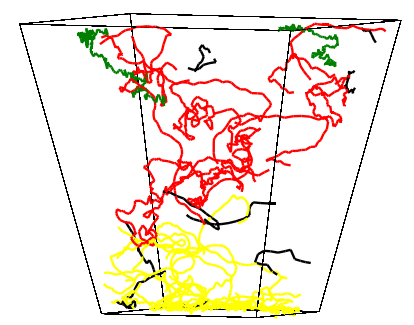

Fig. 7. Complete volume with patterns: Orientation (1, red), Wobbling (2, green), Gyration (3, yellow). The Spinning (4) pattern is not present in this sequence. Patterns which are too short to be classified are plotted in black. See Sequence 1 in the video included as supplemental material.

6. Weeks, E., Crocker, J., Levitt, A., Schofield, A., Weitz, D.: 3d direct imaging of structural relaxation near the colloidal glass transition. Science 287(5452), 627-631 (2000)

7. Berg, H.: Random walks in biology. Princeton University Press, Princeton (1993)

8. Hoyle, D., Rattay, M.: Pca learning for sparse high-dimensional data. Europhysics Letters 62(1) (2003)

9. Wang, X., Grimson, E.: Trajectory analysis and semantic region modeling using a nonparametric bayesian model. In: CVPR (2008)

10. Guyon, I., Weston, J., Barnhill, S., Vapnik, V.: Gene selection for cancer classification using support vector machines. Machine Learning 46(1-3), 389-442 (2004)

11. Sbalzariniy, I., Theriot, J., Koumoutsakos, P.: Machine learning for biological trajectory classification applications. Center for Turbulence Research, 305-316 (2002)

12. Rabiner, L.: A tutorial on hidden markov models and selected applications in speech recognition. Proc. IEEE 77(2) (1989)

13. Chen, M., Kundu, A., Zhou, J.: Off-line handwritten word recognition using a hidden markov model type stochastic network. IEEE Trans. Pattern Anal. Mach. Intell. (TPAMI) 16 (1994)

14. Nefian, A., Hayes, M.H.: Hidden markov models for face recognition. In: ICASSP (1998)

15. Yamato, J., Ohya, J., Ishii, K.: Recognizing human action in time-sequential images using hidden markov model. In: CVPR (1992)

16. Brand, M., Kettnaker, V.: Discovery and segmentation of activities in video. IEEE Trans. Pattern Anal. Mach. Intell (TPAMI) 22(8), 844-851 (2000)

17. Gabor, D.: A new microscopic principle. Nature 161(8), 777 (1948)

18. Xu, W., Jericho, M., Meinertzhagen, I., Kreuzer, H.: Digital in-line holography for biological applications. Proc. Natl. Acad. Sci. U.S.A. 98(20), 11301-11305 (2001)

19. Heydt, M., Divós, P., Grunze, M., Rosenhahn, A.: Analysis of holographic microscopy data to quantitatively investigate three dimensional settlement dynamics of algal zoospores in the vicinity of surfaces. Eur. Phys. J. E (2009)

20. Lu, J., Fugal, J., Nordsiek, H., Saw, E., Shaw, R., Yang, W.: Lagrangian particle tracking in 3d via single-camera in-line digital holography. New Journal of Physics 10 (2008)

21. Leal-Taixé, L., Heydt, M., Rosenhahn, A., Rosenhahn, B.: Automatic tracking of swimming microorganisms in 4d digital in-line holography data. In: IEEE WMVC (2009)

22. Iken, K., Amsler, C., Greer, S., McClintock, J.: Qualitative and quantitative studies of the swimming behaviour of hincksia irregularis (phaeophyceae) spores: ecological implications and parameters for quantitative swimming assays. Phycologia 40, 359-366 (2001)

23. Fugal, J., Schulz, T., Shaw, R.: Practical methods for automated reconstruction and characterization of particles in digital in-line holograms. Meas. Sci. Technol. 20, 075501 (2009) 